\title{
FOTO REATOR SOLAR PARA REMOÇÃO DE POLUENTES E DESINFECÇÃO DE ÁGUA: CRITÉRIOS DE PROJETO E MONTAGEM
}

\author{
L. A. S. CASTRO ${ }^{1}$, R. B. P. MARCELINO ${ }^{1}$, M. C. STARLING $^{1}$, C. C. AMORIM ${ }^{1 *}$, \\ M. M. D. LEÃO ${ }^{1}$ \\ ${ }^{1}$ Universidade Federal de Minas Gerais, Departamento de Engenharia Sanitária e \\ Ambiental \\ *E-mail para contato: camila@desa.ufmg.br
}

\begin{abstract}
RESUMO - O presente trabalho relata a construção de um reator solar em escala piloto do tipo concentrador parabólico composto (CPC) a fim de ser utilizado para o tratamento alternativo de efluentes industriais reais por meio de reações fotocatalíticas solares, do tipo foto-Fenton e fotocatálise com dióxido de titânio $\left(\mathrm{TiO}_{2}\right)$. Nesse sistema, a energia solar é utilizada para aumentar a eficiência da formação dos agentes oxidantes à base de oxigênio, como os radicais hidroxila ( $\left.{ }^{\circ} \mathrm{OH}\right)$, por meio de processos oxidativos avançados. Para a elaboração do projeto do reator CPC os parâmetros de construção: ângulo de aceitação $\left(\theta_{\mathrm{a}}\right)$, fator de concentração da radiação $(\mathrm{Rc})$, material da superfície refletora, tubulação do reator - material, espessura, diâmetro - e ângulo de inclinação do reator foram avaliados e definidos após revisão bibliográfica. Após sua construção e mediante licença de instalação e operação, o foto reator solar foi instalado no terraço da Escola de Engenharia da UFMG. Testes inicias com efluente sintético foram realizados no foto reator para a padronização experimental, com a utilização de um radiômetro para a quantificação da energia solar recebida durante as reações.
\end{abstract}

\section{INTRODUÇÃO}

A utilização de processos convencionais de tratamento de efluentes industriais (processos biológicos e físico-químicos) tem-se mostrado inviável. Além de demandarem longo tempo e grande área para que o efluente atinja aos padrões de lançamento, eles geralmente não provocam a degradação dos contaminantes, mas apenas à transferência de fase dos mesmos.

Neste contexto, os processos oxidativos avançados (POA) têm atraído grande interesse por ser mostrarem mais viáveis, principalmente a longo prazo. Os POA são processos baseados na geração de radicais a base de oxigênio, como os radicais hidroxila $\left({ }^{\bullet} \mathrm{OH}\right)$, agentes altamente oxidantes. Devido ao seu alto potencial padrão de redução, o radical hidroxila é capaz de oxidar uma variedade de compostos orgânicos a $\mathrm{CO}_{2}, \mathrm{H}_{2} \mathrm{O}$ e íons inorgânicos (NOGUEIRA et al., 2007).

O radical hidroxila é geralmente formado em reações que resultam da combinação de oxidantes com irradiação ultravioleta (UV) ou visível e catalisadores, como íons metálicos ou semicondutores (NOGUEIRA et al., 2007). A energia necessária para a 
ocorrência desses processos pode ser um fator limitante para sua aplicação em grande escala, devido aos custos elevados. Logo, a utilização da energia solar para aumentar a eficiência das reações de formação desses radicais mostra-se uma alternativa interessante, tendo em vista a economia de energia promovida e o fato de que a energia solar é limpa e abundante no Brasil (MARTINS et al., 2012).

A fotocatálise solar não só proporciona uma economia de insumos por utilizar uma energia limpa e abundante, mas pode possibilitar, também, a eliminação de compostos tóxicos e/ou recalcitrantes e a desinfecção por microrganismos (MICHAEL et al., 2012). Tanto os processos de fotocatálise como os processos oxidativos avançados solares podem ser economicamente viabilizados se combinados com os processos de tratamento de efluentes e águas já existentes, possibilitando sua utilização por pequenas empresas.

Nesse sentido, tem-se desenvolvido reatores que permitam um melhor aproveitamento da luz solar incidente. Entre os tipos de foto reatores utilizados, a superfície coletora é um dos principais parâmetros que os caracterizam. Ela deve ser avaliada para que seja feita a escolha do equipamento, em função de diferentes eficiências da concentração da radiação incidente sobre o efluente a ser tratado. Segundo Malato et al. (2009), os reatores solares podem ser do tipo coletor não concentrador (CNC), coletor concentrador parabólico (CCP), e coletor parabólico composto (CPC), conforme Figura 1 a seguir.

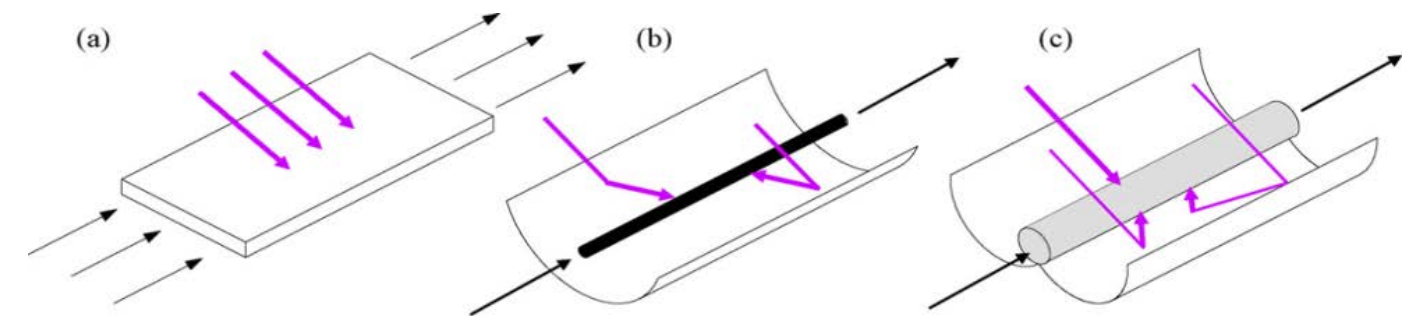

Figura 1 - (1a) Coletor não concentrador, (1b) coletor concentrador parabólico, (1c) coletor parabólico composto. Fonte: Malato et al. (2009).

A Tabela 1 a seguir, relaciona as principais vantagens e desvantagens desses diferentes modelos de foto reatores:

Tabela 1 - Características dos modelos de foto reatores solar

\begin{tabular}{|c|c|c|}
\hline Modelo & Vantagens & Desvantagens \\
\hline $\begin{array}{l}\text { Coletor não } \\
\text { concentrador }\end{array}$ & $\begin{array}{l}\text { - Não concentração da radiação - } \\
\text { diminui a densidade de fótons e a } \\
\text { recombinação das eletro-lacunas, } \\
\text { possibilitando uma maior } \\
\text { eficiência quântica }{ }^{1} \text {; } \\
\text { - Capazes de utilizar a radiação } \\
\text { direta e difusa, e não ocasionam o } \\
\text { aumento excessivo da temperatura } \\
\text { do efluente }{ }^{1} \text {; } \\
\text { - Custos de produção mais baixos - } \\
\text { componentes são mais simples, } \\
\text { resultando em manutenção mais } \\
\text { fácil e de custo reduzido }{ }^{1} \text {. }\end{array}$ & 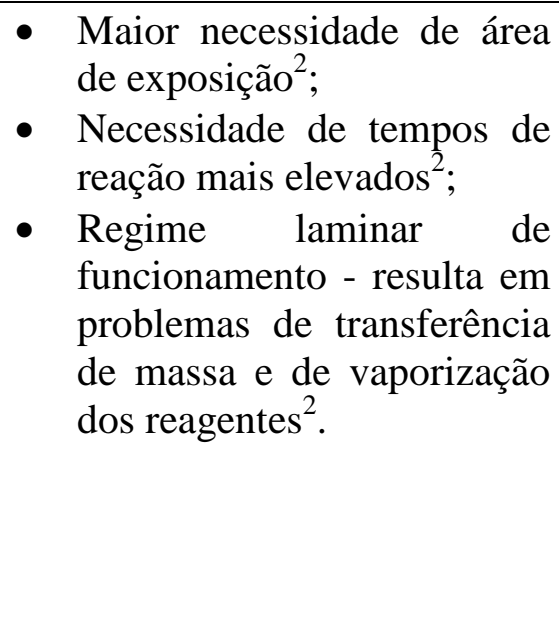 \\
\hline
\end{tabular}




\begin{tabular}{|c|c|c|}
\hline Modelo & Vantagens & Desvantagens \\
\hline $\begin{array}{l}\text { Coletor } \\
\text { concentrador } \\
\text { parabólico }\end{array}$ & $\begin{array}{l}\text { - Ângulo de inclinação ajustável - o } \\
\text { máximo de aproveitamento da } \\
\text { radiação solar; } \\
\text { - Área útil necessária inferior ao } \\
\text { CNC }^{3} \text {; } \\
\text { - Fluxo turbulento - aumenta a } \\
\text { homogeneização de catalisadores } \\
\text { dispersos }{ }^{3} \text {; } \\
\text { - A energia térmica recolhida pode } \\
\text { ser utilizada em outras } \\
\text { aplicações }{ }^{3} \text {. }\end{array}$ & \begin{tabular}{ll} 
- & \multicolumn{2}{l}{ Perda da radiação difusa ${ }^{1}$; } \\
- & Custo elevado de \\
& implantação \\
& manutenção ${ }^{1}$ d \\
- & Menor eficiência quântica ${ }^{1}$; \\
- & Problemas de \\
& superaquecimento da \\
& amostra ${ }^{1}$.
\end{tabular} \\
\hline $\begin{array}{l}\text { Coletor } \\
\text { parabólico } \\
\text { composto }\end{array}$ & 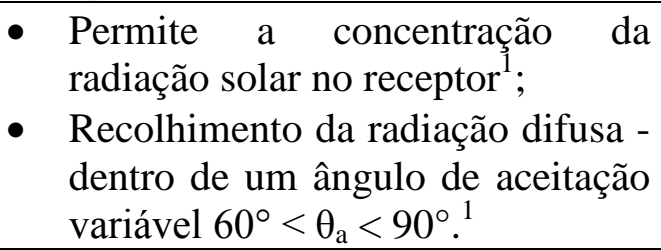 & $\begin{array}{ll}\text { - } & \text { Nenhuma desvantagem } \\
& \text { significativa }^{1} \text {. }\end{array}$ \\
\hline
\end{tabular}

${ }^{1}$ Malato et al. (2009), ${ }^{2}$ Malato et al. (2002), ${ }^{3}$ Munther (2001).

Em comparação ao CCP, nos reatores CPC toda a tubulação de reação sofre influência da radiação incidente - direta e difusa, não apresentando assim zonas escuras. A Figura 2 ilustra essa relação.
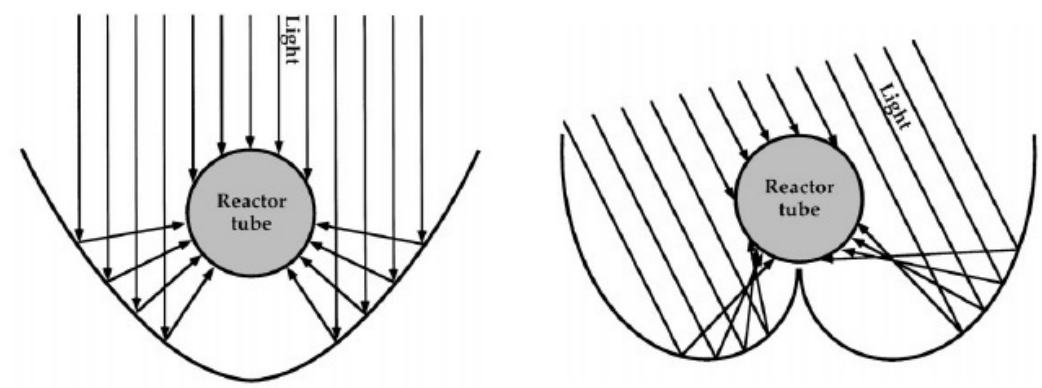

Figura 2: Radiação incidente direta e difusa - à esquerda reator CCP, à direita reator CPC. Fonte: Adaptado de Alfano et al. (2000).

De acordo com Malato et al. (2009), as vantagens dos reatores coletores parabólicos compostos são as mesmas do CNC e CCP e eles não apresentam nenhuma desvantagem significativa. Logo, os CPCs apresentam-se como a melhor opção para processos de fotocatálise solar e foi o modelo de reator adotado nesse estudo.

Embora a tecnologia já esteja disponível e tenha sido aplicada em escala piloto em diferentes processos e efluentes com sua eficácia comprovada, a aplicação prática ainda não é fortemente aceita por potenciais interessados (Tabela 2). Nesse sentido, o desenvolvimento de projetos com envolvimento de potenciais usuários desses reatores são importantes no sentido de reduzir as barreiras à sua implementação (DUARTE et al., 2005). 
Tabela 2 - Utilização de radiação solar em diferentes processos

\begin{tabular}{|c|c|c|c|}
\hline Matriz & Processo & Resultados & Referência \\
\hline $\begin{array}{c}\text { Efluente } \\
\text { farmacêutico - } \\
\text { trimetoprim e } \\
\text { sulfametoxazol }\end{array}$ & $\begin{array}{l}\text { Foto-Fenton solar com } \\
\text { ferrioxalato e } \\
\text { fotocatálise com } \mathrm{TiO}_{2}\end{array}$ & $\begin{array}{l}\text { Remoção dos antibióticos em } \\
\text { todos os processos e mais de } \\
70 \% \text { de mineralização com o } \\
\text { foto-Fenton com ferrioxalato }\end{array}$ & $\begin{array}{l}\text { Dias et } \\
\text { al.(2014) }\end{array}$ \\
\hline $\begin{array}{c}\text { Efluente } \\
\text { farmacêutico }\end{array}$ & $\begin{array}{l}\text { Foto-Fenton solar / } \\
\text { tratamento biológico. }\end{array}$ & $\begin{array}{c}\text { Mais de } 70 \% \text { de mineralização } \\
\text { do contaminante em menos de } 1 \\
\text { hora. }\end{array}$ & $\begin{array}{l}\text { Malato et } \\
\text { al. (2007) }\end{array}$ \\
\hline $\begin{array}{l}\text { Lixiviado de } \\
\text { aterro sanitário } \\
\text { pré-tratado }\end{array}$ & Foto-Fenton solar. & $\begin{array}{l}\text { 60\% de mineralização e 90\% de } \\
\text { redução de aromáticos. }\end{array}$ & $\begin{array}{l}\text { Vilar et al. } \\
\text { (2011) }\end{array}$ \\
\hline Efluente têxtil & $\begin{array}{l}\text { Foto-Fenton solar, } \\
\mathrm{TiO}_{2} / \mathrm{UV}, \mathrm{H}_{2} \mathrm{O}_{2} / \mathrm{UV} \text {. }\end{array}$ & $\begin{array}{l}\text { 30\% e } 36 \% \text { de mineralização } \\
\text { para } \mathrm{TiO}_{2} / \mathrm{UV} \text { e } \mathrm{H}_{2} \mathrm{O}_{2} / \mathrm{UV} \text {, } \\
\text { respectivamente. } 89 \% \text { de } \\
\text { mineralização para foto-Fenton } \\
\text { solar. }\end{array}$ & $\begin{array}{l}\text { Vilar et al. } \\
\text { (2011) }\end{array}$ \\
\hline $\begin{array}{l}\text { Corante têxtil } \\
\text { Reactive Blue } \\
\quad 4\end{array}$ & $\begin{array}{l}\text { Foto-Fenton solar, } \\
\text { foto-Fenton solar com } \\
\text { ferrioxalato. }\end{array}$ & $\begin{array}{l}\text { 100\% remoção de cor para } \\
\text { ambos, e } 83 \% \text { e } 100 \% \text { de } \\
\text { remoção de DQO para foto- } \\
\text { Fenton e foto-Fenton com } \\
\text { ferrioxalato, respectivamente. }\end{array}$ & $\begin{array}{l}\text { Durán et } \\
\text { al. (2008) }\end{array}$ \\
\hline
\end{tabular}

Dessa forma, o presente trabalho tem por objetivo a construção de um foto reator solar CPC para posterior aplicação no tratamento de efluente gerado em uma indústria têxtil da região metropolitana de Belo Horizonte, dentre outros. Além da definição dos critérios para a construção do reator, foram estudados e apresentados resultados iniciais obtidos da degradação de um efluente sintético que se constituiu de um corante utilizado na indústria em questão. Os experimentos foram feitos para avaliar o funcionamento do equipamento e nortear experimentos futuros.

\section{MATERIAL E MÉTODOS}

Para a elaboração do projeto do reator, os parâmetros: ângulo de aceitação $\left(\theta_{a}\right)$, fator de concentração da radiação (Rc), material e dimensões da superfície refletora, tubulação do reator - material, espessura e diâmetro, regime de escoamento e ângulo de inclinação do reator foram avaliados e definidos após revisão bibliográfica.

A escolha do local de instalação do reator foi feita com base na incidência de radiação solar, possibilidade de acesso e distância dos laboratórios do Departamento de Engenharia Sanitária e Ambiental.

O corante utilizado nos experimentos foi o Reactive Yellow 145, que possui absorção máxima na região do visível igual a $420 \mathrm{~nm}(\lambda=420 \mathrm{~nm})$. O efluente sintético foi produzido com concentração de $50,0 \mathrm{mg} / \mathrm{L}$ de corante. A partir daí, foram realizados três processos com radiação solar: $\mathrm{TiO}_{2}, \mathrm{TiO}_{2} / \mathrm{H}_{2} \mathrm{O}_{2}$, foto-Fenton com ferrioxalato. Nos experimentos com $\mathrm{TiO}_{2}$ utilizou-se 200,0 mg/L de dióxido de titânio (P-25), contendo um deles também 200,0 mg/L de $\mathrm{H}_{2} \mathrm{O}_{2}$. Para a reação de foto-Fenton foram utilizados $20 \mathrm{mg} / \mathrm{L}$ de $\mathrm{Fe}^{2+}$, pela adição de $\mathrm{FeSO}_{4} .7 \mathrm{H}_{2} \mathrm{O}$ (sólido), e $80 \mathrm{mg} / \mathrm{L}$ de ácido oxálico, para que houvesse a formação do complexo ferrioxalato, entre o $\mathrm{Fe}^{3+}$ e o ácido oxálico. $\mathrm{O}$ pH 
utilizado foi o natural da solução, igual a 6,76. Entretanto para a reação de foto-Fenton com ferrioxalato o $\mathrm{pH}$ foi ajustado inicialmente para 5,0 com ácido sulfúrico. As concentrações de reagente utilizadas foram baseadas em revisão de literatura.

As amostras coletadas nos tempos 0', 5', 10', 15’, 30', 45', 60', 120' e 180' foram filtradas em filtro de nitrocelulose $0,45 \mu \mathrm{m}$ e analisadas em função do carbono orgânico dissolvido (COD; mg/L) por meio de um Analisador de Carbono Orgânico Total (Shimadzu) e da cor (absorbância) por meio de varredura nos comprimentos de onda do espectro visível por um especfotômetro (Lambda XLS). Nos casos de utilização de $\mathrm{H}_{2} \mathrm{O}_{2}$, o peróxido residual foi determinado pelo método do metavanadato. Foi monitorada a temperatura do efluente que atingiu no máximo $50,0^{\circ} \mathrm{C}$.

A radiação incidente foi obtida por meio de um radiômetro (CUV 5 Kipp \& Zonen) e um datalogger (METEON Kipp \& Zonen), com intervalo de leitura da radiação incidente a cada cinco minutos. A Equação 1 permite obter a quantidade de energia UV acumulada (Quv) recebida na superfície de reação por intervalo do volume de água no interior do reator, no intervalo de tempo t, segundo Vilar et al. (2011):

$$
Q_{U V, n}=Q_{U V, n-1}+\Delta t_{n} \overline{U V}_{G, n} \frac{A_{r}}{V_{t}}
$$

Onde $\Delta \mathrm{t}_{\mathrm{n}}$ corresponde ao intervalo de tempo, $\mathrm{V}_{\mathrm{t}}$ o total do volume do reator $(\mathrm{L})$, $\mathrm{A}_{\mathrm{r}}$ a área da superfície do coletor iluminado $\left(\mathrm{m}^{2}\right)$, e $\overline{U V}_{G, n}$ a radiação média solar ultravioleta $\left(\mathrm{W} \mathrm{m}^{-2}\right)$.

\section{RESULTADOS E DISCUSSÃO}

A tubulação foi construída com vidro borossilicato com baixo teor de ferro, pois esse material apresenta boa resistência química e transmissão da radiação UV na gama solar, além de apresentar custos reduzidos.

Foram utilizados cinco tubos de 1,20 m cada, com diâmetro externo (d) igual a 50 mm e espessura da parede (e) de 1,8 mm. A escolha do diâmetro foi realizada buscandose um valor médio a partir do qual seria possível a penetração da radiação por efluentes de diferentes características. As conexões e demais tubulações utilizadas são de PVC, devido ao baixo custo e facilidade de obtenção das peças.

O material adotado para a confecção da superfície refletora foi o alumínio, devido ao seu baixo custo e elevada refletividade no espectro UV solar. A partir da relação (Equação 2) proposta por Malato et al. (2009), a abertura da parábola da superfície refletora (A), pode ser obtida:

$$
R_{C, C P C}=\frac{1}{\operatorname{sen} \theta_{a}}=\frac{A}{2 \pi r}
$$

Um caso especial é obtido quando o ângulo de aceitação $\left(\theta_{\mathrm{a}}\right)$ é igual a $90^{\circ}$, ocasionando um fator de concentração da radiação igual a 1, proporcionando que a totalidade da radiação UV que atinge o CPC possa ser coletada e direcionada para o reator (MALATO et al., 2009). Dessa forma, a luz projetada seria a mesma que incidiria sobre um reator de placa plana. A figura 3 a seguir, ilustra a superfície refletora. Para a configuração proposta a abertura A assume valor igual a 145,8 mm. 


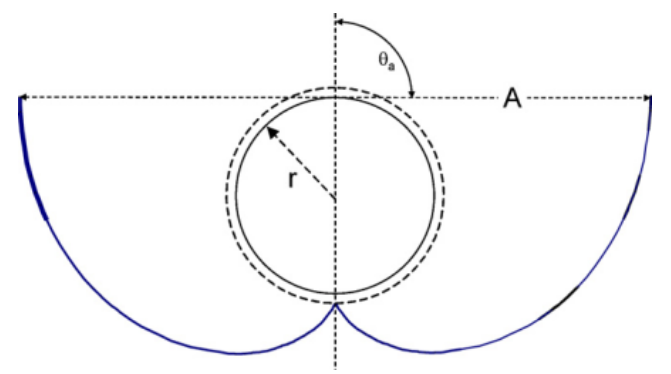

Figura 3 - Configuração da parábola da superfície refletora. Fonte: Malato et al. (2009).

O regime hidráulico de escoamento deve ser o turbulento, a fim de evitar a deposição de compostos nas paredes do equipamento e possibilitar a homogeneização eficiente dos reagentes com o efluente a ser tratado. Dimensionou-se, assim, uma bomba cujo fluxo ótimo foi estimado em 13L/min.

O ângulo de inclinação vertical da superfície que recebe a radiação deve ser igual à latitude do local de instalação, de forma que a radiação incida segundo o ângulo de $90^{\circ}$, proporcionando o máximo de aproveitamento da radiação. Sendo assim, foi adotado o valor de $20^{\circ}$, correspondente à latitude da cidade de Belo Horizonte. O mesmo ângulo de $20^{\circ}$ foi adotado para o radiômetro, a fim de que a leitura da radiação incidente seja a mesma dos tubos de reação. O reator possui também uma inclinação horizontal de $1^{\circ}$ para evitar o acúmulo de água de chuva na estrutura do equipamento.

A Figura 4 apresenta o equipamento. O volume total irradiado corresponde a aproximadamente 10 litros, e a adoção de um by-pass, com duas bombas e dois tanques independentes, possibilita utilizar o reator com dois e três tubos de reação, correspondendo a 4 e 6 litros respectivamente, permitindo tratar dois efluentes ao mesmo tempo.
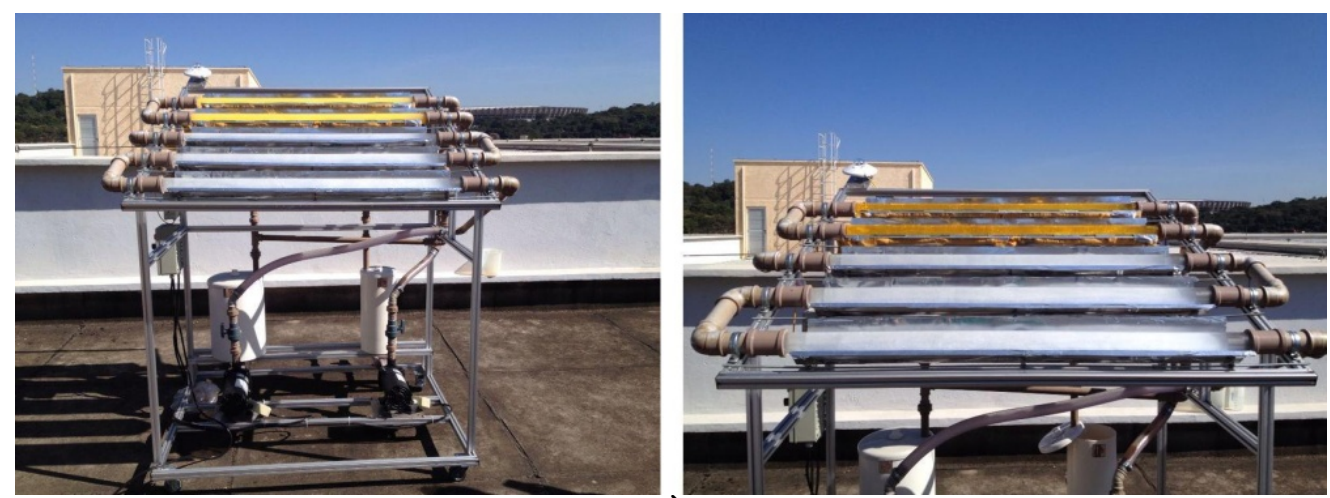

Figura 4 - Instalação do reator solar CPC. À esquerda vista geral, à direita enfoque na superfície de reação.

Na Figura 5 estão apresentadas as concentrações de COD e absorbância da cor no comprimento de onda de $420 \mathrm{~nm}$ característico do corante utilizado (Figura 5a e 5b respectivamente). Pode ser observado que os três processos avaliados foram eficientes na remoção de cor do efluente, sendo que o processo de foto-Fenton com ferrioxalato conseguiu remover a cor com uma menor radiação acumulada e, consequentemente, um menor tempo de reação. 

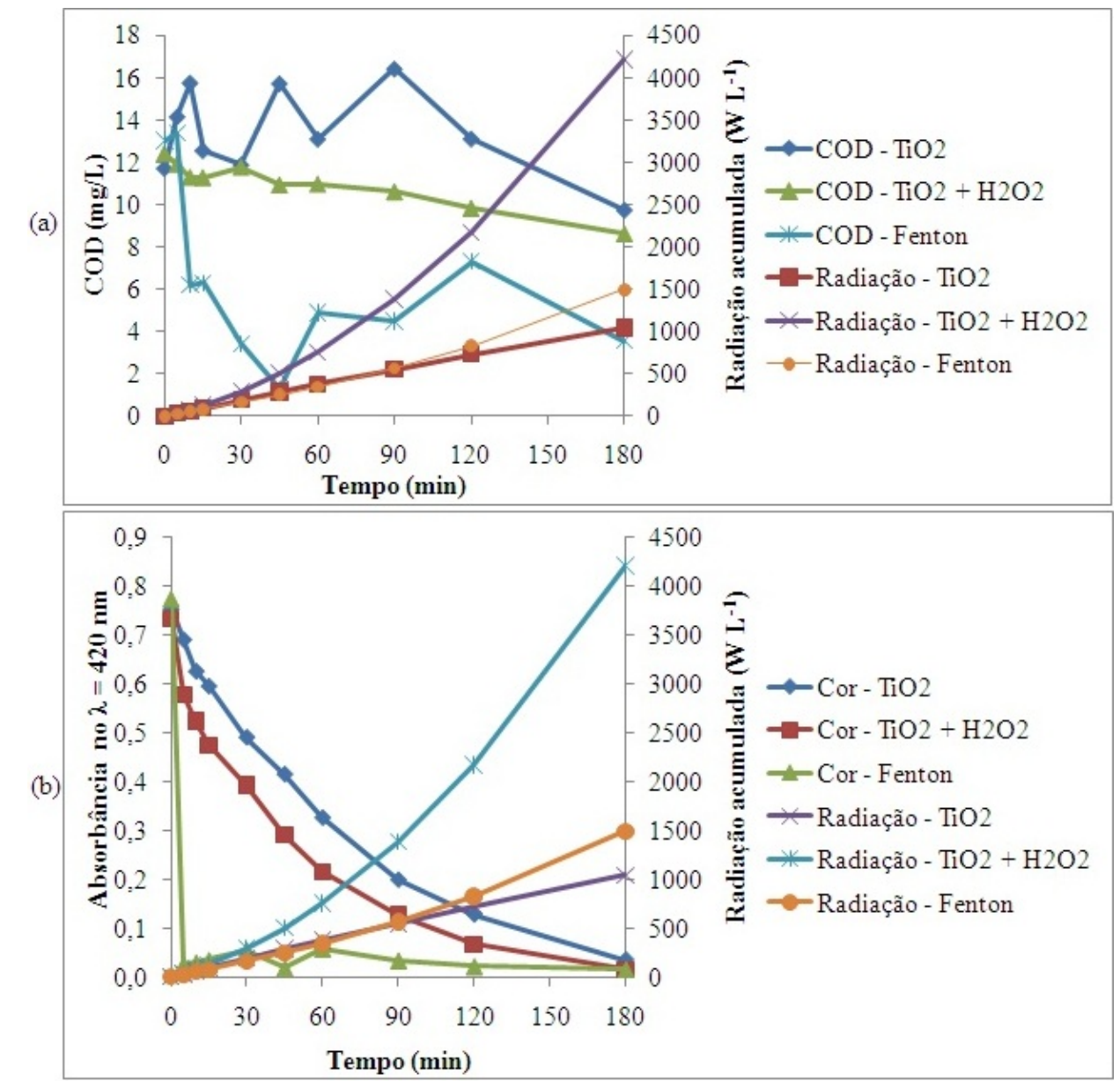

Figura 5 - (5a) Concentração de COD $\left(\mathrm{mg} \mathrm{L}^{-1}\right)$ e radiação acumulada $\left(\mathrm{W} \mathrm{L}^{-1}\right)$ ao longo do tempo (min); (5b) absorbância em $420 \mathrm{~nm}$ e radiação acumulada ( $\mathrm{W} \mathrm{L}^{-1}$ ) ao longo do tempo (min).

Em relação à remoção de COD, o foto-Fenton ferrioxalato se mostrou mais eficiente (remoção de aproximadamente 73\%) do que os demais processos. Vale ressaltar que os processos fotocatalíticos para a geração de radicais hidroxila possuem um baixo rendimento quântico (a taxa de uma reação induzida pela absorção de fótons dividida pelo fluxo de fótons absorvidos) $4 \pm 0,3 \%$ - para a geração de ${ }^{\bullet} \mathrm{OH}$ pela fotocatálise com $\mathrm{TiO}_{2}$ (SUN \& BOLTON, 1996) (MARCELINO et al., 2014). A maioria dos fótons absorvidos é perdida para a recombinação das eletro-lacunas.

\section{CONCLUSÕES}

A aplicação de foto-reator solar é uma alternativa promissora no tratamento de efluentes recalcitrantes, pois a utilização da energia solar, limpa e sem ônus, representa uma economia de gastos de operação. A escolha correta dos parâmetros listados possibilita melhor eficiência de funcionamento, podendo o equipamento substituir os tratamentos convencionais ou complementá-los. No estudo, o foto-Fenton apresentou maior remoção de cor com menor intensidade de radiação recebida, tornando-o uma alternativa promissora. Isso pode possibilitar a reutilização de um efluente clarificado na planta industrial ou adequação aos parâmetros de lançamento. 


\section{AGRADECIMENTOS}

Os autores agradecem ao CNPq, a Capes e à FAPEMIG pelos auxílios financeiros concedidos.

\section{REFERÊNCIAS BIBLIOGRÁFICAS}

ALFANO, O. M.; BAHNEMANN, D.; CASSANO, A. E.; DILLERT, R.; GOSLICH, R. Photocatalysis in water environments using artificial and solar light. Catalysis Today, v. 58, p. 199-230, 2000.

DIAS, I. N.; SOUZA, B. S.; PEREIRA, J. H. O. S.; MOREIRA, F. C.; DEZOTTI, M.; BOAVENTURA, R. A. R.; VILAR, V. J. P. Enhancement of the photo-Fenton reaction at near neutral $\mathrm{pH}$ through the use of ferrioxalate complexes: A case study on trimethoprim and sulfamethoxazole antibiotics removal from aqueous solutions. Chemical Engineering Journal, v. 247, p. 302-313, 2014.

DUARTE, E. T. F. M.; XAVIER, T. P.; SOUZA, D. R. D.; MIRANDA, J. A. D.; MACHADO, A. E. D. H.; JUNG, C.; OLIVEIRA, L. D.; SATTLER, C. Construção e estudos de perfomance de um reator fotoquímico tipo CPC ("Compound Parabolic Concentrator"). Química Nova, v. 28, p. 921-926, 2005.

DURÁN, A.; MONTEAGUDO, J. M.; AMORES, E. Solar photo-Fenton degradation of Reactive Blue 4 in a CPC reactor. Applied Catalysis B: Environmental, v. 80, p. 42-50, 2008.

MALATO, S.; BLANCO, J.; MALDONADO, M. I.; OLLER, I.; GERNJAK, W.; PÉREZ-ESTRADA, L. Coupling solar photo-Fenton and biotreatment at industrial scale: Main results of a demonstration plant. Journal of Hazardous Materials, v. 146, p. 440-446, 2007.

MALATO, S.; BLANCO, J.; VIDAL, A.; RICHTER, C. Photocatalysis with solar energy at a pilot-plant scale: an overview. Applied Catalysis B: Environmental, v. 37, p. 1-15, 2002.

MALATO, S.; FERNÁNDEZ-IBÁÑEZ, P.; MALDONADO, M. I.; BLANCO, J.; GERNJAK, W. Decontamination and disinfection of water by solar photocatalysis: Recent overview and trends. Catalysis Today, v. 147, p. 1-59, 2009.

MARCELINO, R. B. P.; QUEIROZ, M. T. A.; AMORIM, C. C.; LEÃO, M. M. D.; BRITES-NÓBREGA, F. F. Solar energy for wastewater treatment: review of international technologies and their applicability in Brazil. Environmental Science and Pollution Research, p. 1-12, 2014.

MARTINS, F. R.; ABREU, S. L.; PEREIRA, E. B. Scenarios for solar thermal energy applications in Brazil. Energy Policy, v. 48, p. 640-649, 2012. 
MICHAEL, I.; HAPESHI, E.; MICHAEL, C.; VARELA, A. R.; KYRIAKOU, S.; MANAIA, C. M.; FATTA-KASSINOS, D. Solar photo-Fenton process on the abatement of antibiotics at a pilot scale: Degradation kinetics, ecotoxicity and phytotoxicity assessment and removal of antibiotic resistant enterococci. Water Research, v. 46, p. 5621-5634, 2012.

MUNTHER, R. Advanced Oxidation Processes - Current status and prospects. Proceedings of the Estonian Academy of Sciences, Chemistry (2001) 50:59-80.

NOGUEIRA, R. F. P.; TROVÓ, A. G.; SILVA, M. R. A. D.; VILLA, R. D.; OLIVEIRA, M. C. D. Fundamentos e aplicações ambientais dos processos fenton e foto-fenton. Química Nova, v. 30, p. 400-408, 2007.

SUN, L; BOLTON, J. R. (1996): Determination of the Quantum Yield for the Photochemical Generation of Hydroxyl Radicals in TiO2 Suspensions. The Journal of Physical Chemistry 100, 4127-4134.

VILAR, V. J. P.; CAPELO, S. M. S.; SILVA, T. F. C. V.; BOAVENTURA, R. A. R. Solar photo-Fenton as a pre-oxidation step for biological treatment of landfill leachate in a pilot plant with CPCs. Catalysis Today, v. 161, p. 228-234, 2011.

VILAR, V. J. P.; PINHO, L. X.; PINTOR, A. M. A.; BOAVENTURA, R. A. R. Treatment of textile wastewaters by solar-driven advanced oxidation processes. Solar Energy, v. 85, p. 1927-1934, 2011, 2011. 